\title{
Reconstruction Techniques in Supersymmetry Searches
}

Mark Hodgkinson, on behalf of the ATLAS Collaboration*

University of Sheffield

E-mail: m.hodgkinson@sheffield.ac.uk

\begin{abstract}
Many supersymmetric scenarios feature final states with non-standard final state objects. The production of massive sparticles can lead to the production of boosted top quarks or vector bosons, high-pt b-jets. At the same time, transitions between nearly mass-degenerate sparticles can challenge the standard reconstruction because of the presence of very soft leptons or jets. The talk will review the application of innovative reconstruction techniques to supersymmetry searches in ATLAS.
\end{abstract}

The 39th International Conference on High Energy Physics (ICHEP2018)

4-11 July, 2018

Seoul, Korea

${ }^{*}$ Speaker. 


\section{Introduction}

The ATLAS detector [1] had collected $108 \mathrm{fb}^{-1}$ by the end of July 2018 in LHC Run 2. The main changes to the detector configuration for Run 2 are the addition of the insertable B-Layer (IBL) which provides improved vertexing capabilities. The supersymmetry framework provides a wide range of models covering a large kinematic phase space. In the example of gluino $(\tilde{g})$ production, with subsequent decays into two light quarks and a neutralino $\left(\tilde{\chi}_{1}^{0}\right)$, if the mass difference between the $\tilde{g}$ and $\tilde{\chi}_{1}^{0}$ is large then two jets become boosted such that they are observed as one large radius reconstructed jet. Conversely if the mass difference is small then a low $p_{\mathrm{T}}$ jet is produced. Similar arguments apply to topologies involving lepton production, and in particular we will subsequently discuss reconstruction of low $p_{\mathrm{T}}$ (soft) leptons.

\section{Reconstruction of Soft Leptons}

Electrons are currently reconstructed down to $4.5 \mathrm{GeV}$. The electron reconstruction matches the calorimeter extrapolated track coordinates (corrected for Bremsstrahlung) to a seed calorimeter topological cluster (topocluster). Nearby topoclusters matched in a fixed sized window are also associated to the electron candidate. This improves electron resolution because this better (than the old fixed size sliding window clustering algorithm) captures Bremsstrahlung photons emitted in the phi direction. Further improvements are expected because the topoclusters, formed based on noise thresholds (unlike the old style fixed size sliding window clusters), allow one to go down to lower $p_{\mathrm{T}}$ thresholds. Figure 1a shows the efficiency of electron reconstruction and identification in $Z \rightarrow e e$ events as a function of transverse energy. There are three categories of muons reconstructed which

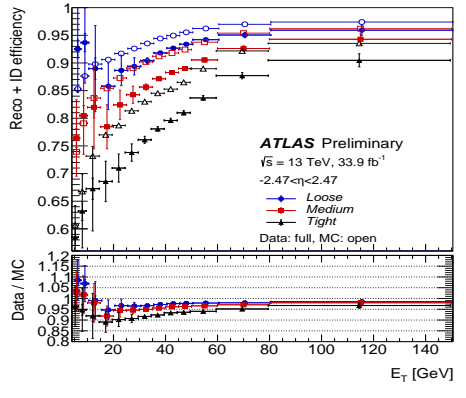

(a)

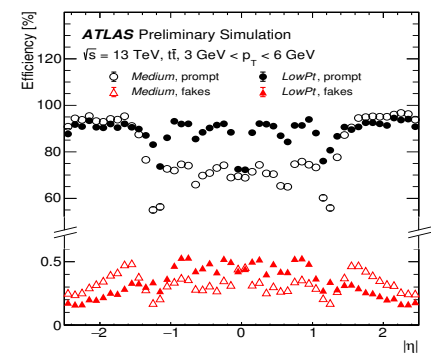

(b)

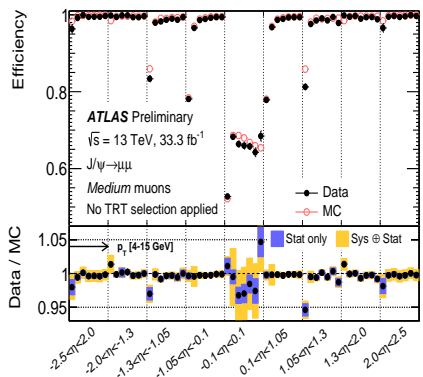

(c)

Figure 1: (a) Efficiency of electron reconstruction and identification in $Z \rightarrow e e$ events as a function of transverse energy [2], (b) Efficiency of muons fulfilling the LowPt and Medium working points in simulated $t \bar{t}$ events [3] and (c) Efficiency of muons fulfilling the Medium working point in different $p_{\mathrm{T}}$ bins for each $\eta$ region, using a $J / \psi \rightarrow \mu \mu$ selection in data [4].

involve combining the Inner Detector track with a Muon Spectrometer (MS) track, muon segments or calorimeter minimum ionising particle deposit. A fourth category requires the MS track to have a loose compatibility with the interaction point. The reconstruction algorithms can currently go down to $p_{\mathrm{T}}$ of $3 \mathrm{GeV}$ (the average energy loss in the calorimeter is around $3 \mathrm{GeV}$ ). The challenges to achieve this include improving the reconstruction algorithms using only the hits from the innermost muon station whilst keeping the fake rate under control, as well as commissioning of a new partial event building trigger to select 1 muon and 1 track signatures. Current published SUSY analyses use muons down to $4 \mathrm{GeV}$, 
Analyses targeting $\tilde{g}$ decays via sleptons $(\tilde{l})$ with $7 \mathrm{GeV} p_{\mathrm{T}}$ lepton thresholds show significant gains in sensitivity (Figure 2a). Using the (then) lowest supported lepton thresholds of $4 \mathrm{GeV}$ muons and $5 \mathrm{GeV}$ electrons was the key to constraining models with $\tilde{l}-\tilde{\chi}_{1}^{0}$ mass differences down to $1 \mathrm{GeV}$ (Figure 2b).

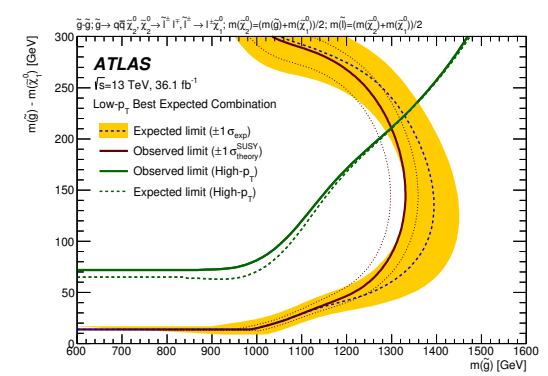

(a)

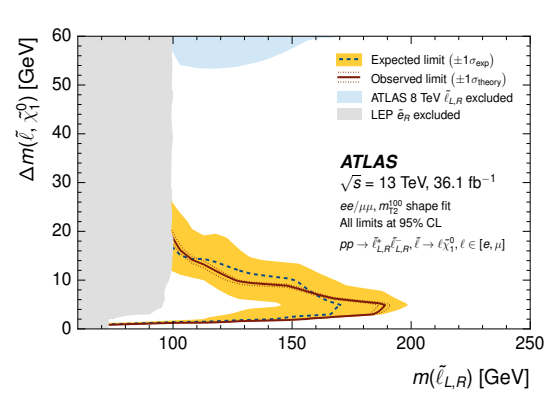

(b)

Figure 2: (a) Exclusion in the plane of the $\tilde{g}$ mass vs. $\tilde{g}-\tilde{\chi}_{1}^{0}$ mass difference [5] and (b) Exclusion in the plane of the $\tilde{l}$ mass vs. $\tilde{l}-\tilde{\chi}_{1}^{0}$ mass difference [6].

\section{Large Radius Jets}

The "standard" jet finding in ATLAS uses the anti- $k_{t}$ algorithm with a radius parameter of 0.4. In boosted topologies jets with a larger radius parameter are used. One can either run jet finding with a large radius parameter or cluster the existing calibrated 0.4 jets into a larger jet (known as reclustering). The advantage of reclustering is that one can use the already known calibrations and uncertainties derived for the 0.4 jets, which allows more flexibility because no dedicated calibration is needed for the large radius jets. The mass resolution from both techniques is shown in Figure $3 \mathrm{a}$.

In the production of stop $(\tilde{t})$ particles, which decay into top $(t)$ and $\tilde{\chi}_{1}^{0}$ we can tag hadronic top decays in order to suppress the top pair background process. We recluster 0.4 jets into 3.0 jets and then reduce the jet radius assuming that $R\left(p_{\mathrm{T}}\right)=2 m_{t} / p_{\mathrm{T}}$. If this results in a large change in the jet $p_{\mathrm{T}}$ then the jet is discarded. Finally one can place a cut on the reclustered jet mass, the distribution of which for data and MC is shown in Figure 3b. Large radius jets can also be used to construct simple kinematic quantities that differentiate signal and background, as shown in Figure $3 \mathrm{c}$ - for example when searching for production of $\tilde{g}$ 's that decay into $t$ and a $\tilde{\chi}_{1}^{0}$ we sum the mass of all reclustered 0.8 jets which is typically large in models producing four $t$, whilst being small for the Standard Model (SM) backgrounds.

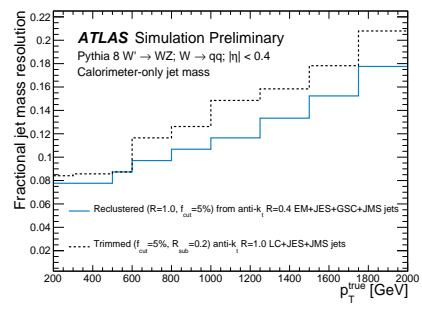

(a)

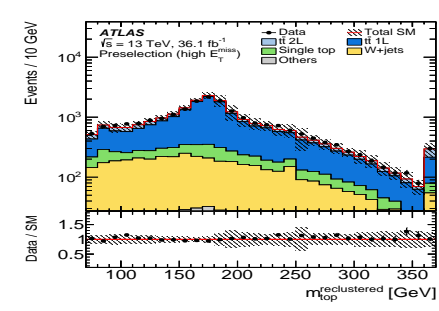

(b)

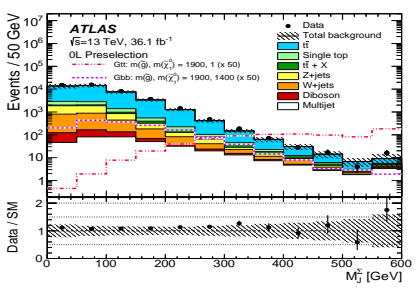

(c)

Figure 3: (a) Comparison of mass resolution for large radius jet finding and reclustered jet finding [7], (b) reclustered top mass [8] and (c) sum of the mass of large radius jets [9]. 


\section{Flavour Tagging}

Given many signatures involve decays to bottom or charm quarks, ATLAS has developed algorithms to tag both bottom and charm jets. To tag bottom quark initiated jets we use a Boosted Decision Tree which analyses the output of the Impact Parameter, Secondary Vertex Finding and Decay Chain Multi-Vertex Algorithms. Figure 4a illustrates significant improvements with respect to the performance seen in Run 1 illustrated with the $77 \%$ fixed efficiency working point. A Charm tagging algorithm makes use of additional variables related to the different kinematics of charm and bottom hadron decays (such as the invariant mass of secondary tracks, secondary track rapidities, distance from primary to secondary vertex, fraction of jet energy carried secondary tracks etc). Figure $4 \mathrm{~b}$ shows the light and $\mathrm{b}$-jet rejection for this algorithm. The exclusion obtained in terms of the scharm $(\tilde{c})$ mass and other parameters, when using this algorithm, is shown in Figure 4c.

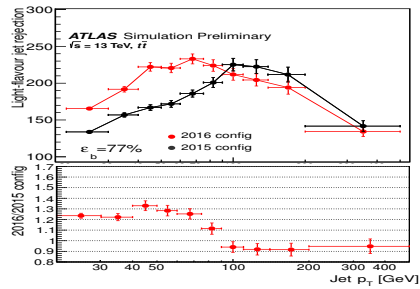

(a)

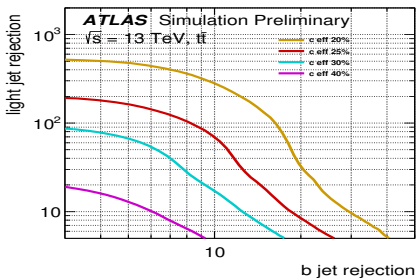

(b)

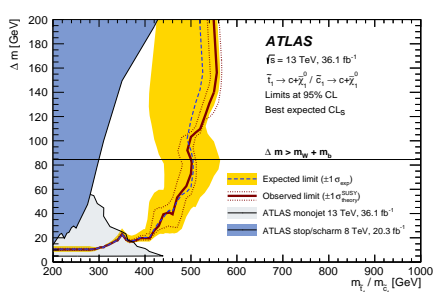

(c)

Figure 4: (a) Comparison of light rejection in ATLAS Run 1 and Run 2 [10], (b) Light and b-jet rejection for the charm-tagging algorithm in top pair events [11] and (c) Exclusion in the plane of the $\tilde{t}$ or $\tilde{c}$ mass and mass difference [12].

\section{Conclusions}

Reconstruction algorithms are helping push the sensitivity of data analyses into more extreme parts of the supersymmetry phase space. New ideas are being investigated in ATLAS such as Particle Flow, low $p_{\mathrm{T}} \mathrm{b}$-tagging with track jets and machine learning. More results are to come from ATLAS, some of which will take advantage of the best performing new reconstruction techniques.

\section{References}

[1] ATLAS Collaboration, 2008 JINST 3 S08003

[2] ATLAS Collaboration, ATLAS-EGAM-2017-003

[3] ATLAS Collaboration, ATLAS-MUON-2018-005

[4] ATLAS Collaboration, ATLAS-MUON-2017-001

[5] ATLAS Collaboration, Submitted to EPJC, arxiv:1805.11381

[6] ATLAS Collaboration, Phys. Rev. D 97 (2018) 052010

[7] ATLAS Collaboration, ATLAS-CONF-2017-062

[8] ATLAS Collaboration, JHEP 06 (2018) 108

[9] ATLAS Collaboration, JHEP 06 (2018) 107

[10] ATLAS Collaboration, ATLAS-PHYS-PUB-2016-012

[11] ATLAS Collaboration, ATLAS-PHYS-PUB-2017-013

[12] ATLAS Collaboration, JHEP 09 (2018) 050 